\title{
Corrections in the Chemical Literature: Their Number and Nature
}

\author{
David E. Hubbard \\ Texas A\&M University Libraries, College Station, Texas
}

\begin{abstract}
Corrections, errata, and corrigenda have played a vital role in maintaining the integrity of the scholarly journal record. Being cognizant of these corrections has always been a challenge for researchers and their management a bane to librarians. Identification of corrections has been made easier with the indexing of corrections by a few commercial databases and more recently by some publishers linking corrections to articles on their e-journal platforms. Few studies have examined the nature of these corrections, especially outside of the biomedical literature where article retraction has been the primary focus. This paper quantifies and qualifies the nature of corrections within the field of chemistry and compares the effectiveness of Scopus and Web of Science in locating corrections within scholarly journals. The study found that the correction rate averaged about 1.4 percent for the journals examined. While there were numerous types of corrections, chemical structures, omission of relevant references, and data errors were some of the most frequent types of published corrections.
\end{abstract}

KEYWORDS corrigendum, errors, erratum, scientific literature

\section{INTRODUCTION}

Corrections, also referred to as errata and corrigenda, have played a crucial role in

maintaining the integrity of the scholarly journals since the printing of the first scientific journals in the mid-1600s. While there has been considerable interest in the issue of retraction of journal articles within the biomedical literature, there appears to be little research into the nature of corrections outside the biomedical literature.

Articles are occasionally retracted for scientific misconduct or fraud, though more often a retraction is due to unintentional mistakes (Budd et al. 1999; Nath, Marcus, and Druss 2006; Redman, Yarandi, and Merz 2008). Corrections, on the other hand, are published for a variety of reasons and are generally not associated with fraud or misconduct. Published corrections may 
range from benign typographic errors to invalid conclusions. When an error is discovered in a journal article, authors, editors and/or publishers often publish a correction in a subsequent issue. While corrections may not appear as significant as retractions, their impact may be just as detrimental as errors or research misconduct in a retracted article. Journal policies and author decisions regarding what should be retracted and what is merely corrected are not always clear. One author may choose to retract an article containing major errors, whereas another author may choose to publish a correction. Even in cases of research misconduct or fraud, journal policies or author instructions do not typically address or outline a process for submitting a retraction (Atlas 2004).

Awareness of the number and nature of these corrections can help researchers prevent such errors in their published work by understanding the most common errors. Understanding how to identify and locate corrections is essential to understanding articles, reproducing experiments, and reducing error transmission to other articles and books. This paper seeks to quantify and qualify the nature of corrections within the chemical literature, which can pose a unique set of challenges compared to other scientific and technical literature due to chemical nomenclature, chemical formulas, and chemical structures.

Loepprich (1973) and Kotzin and Schuyler (1989) conducted some of the few studies that examined the nature of corrections in terms of error type; however, their focus was on the biomedical literature. Loepprich categorized 894 errata notices from 274 journals during a sixmonth period into six broad categories of errors: (1) topographical, figures, illustrations, and legends; (2) errors in text, typographical errors, and pagination errors; (3) formulas, equations, and dosages; (4) bibliographical errors, titles, authors, and citations; (5) news releases, future meetings, death notices, etc.; and (6) not defined. Kotzin and Schuyler (1989) examined 1987 
and 1988 corrections added to the Medline database and categorized the corrections into seven categories based on the Medline field where the correction occurred: (1) authors, (2) titles, (3) abstracts, (4) text, (5) tables, (6) figures, and (7) other. While locations of the corrections were identified and quantified, the types of corrections were not characterized beyond their location. Two studies that characterized corrections within chemistry journals are Addelston and Goldsmith (1966) and Sabine (1985). Addelston and Goldsmith (1966) examined corrections in the Journal of the American Chemical Society and the Journal of Organic Chemistry from 1964 to 1965 . The authors identified 21 types of errors but did not attempt to quantify and categorize all corrections. Of the 157 corrections examined, errors associated with structural diagrams, numerical data, and mathematical equations were the most common. Sabine (1985) selected biological publications based on his "own scientific bias" (p. 63) but included the Journal of the American Chemical Society. He examined 10 journals from 1983 and employed 5 loosely defined categories for the corrections found: (1) marginal errors, (2) social errors, (3) minor errors, (4) major errors, and (5) retractions. Sabine defined and provided several examples of each type of error. He did not attempt to quantify each type of error but stated that about onethird were either major errors or retractions. With respect to the Journal of the American Chemical Society, Sabine found 46 published corrections among 1,797 articles. He then computed an error rate of 2.6 percent for the Journal of the American Chemical Society and 2.3 percent overall for the ten journals studied. The methodology for both studies involved consulting the actual print journals to locate corrections.

Locating corrections became much easier when Medline and Web of Science began indexing corrections. Medline began indexing corrections in 1987 (Colaianni 1992) and Web of Science (i.e., Science Citation Index) since its inception (Garfield 1963). Garfield (1987) had even 
referred to the Science Citation Index as a correction index and urged researchers to check the literature for corrections and retractions. More recently, Elsevier launched Scopus and it also indexes corrections. Medline maintains separate Publication Types for indexing of retractions and corrections, while Scopus and Web of Science include retractions as Erratum and Correction, respectively.

The advent of the Web has also allowed publishers to link a correction to the original article on their e-journal platforms. Poworoznek (2003), however, has shown that many publishers have failed to provide such linkages. Of the forty-three physical science journals Poworoznek studied, only twenty-six had links to corrections within their e-journal platforms. Similarly, Jones, Watson, Comegys, Burnett, and Tucker (2003) found a lack of standardization in how publishers of biomedical journals were addressing corrections through linking on their e-journal platforms.

Considering that corrections are often temporally and spatially separated, one may ask how effective published corrections are at correcting the scholarly record. One of the few studies that examined error transmission and effectiveness of corrections within the chemical literature was by Devlin (1969). Devlin found that of 528 papers citing articles with associated errata sheets, 518 neither repeated nor corrected the error because the authors cited unaffected parts of the original papers. Only ten papers corrected the errors. While Devlin concluded that errata sheets were effective at preventing error transmission, numerous studies involving retractions (a type of correction) in the biomedical literature have shown that many retracted papers continue to be cited after being retracted (e.g., Budd et al. 1999; Pfeifer and Snodgrass 1990; Redman, Yarandi, and Merz 2008), though often with decreased frequency over time (Garfield and Welljams-Dorof 1990). This study will provide more in-depth quantification and qualification of corrections within the top multidisciplinary chemistry journals compared to the two earlier studies conducted 
over twenty years ago. This study will not only serve as an update, but will also investigate quantitative and qualitative changes with respect to published corrections. Another objective of the study is to evaluate the effectiveness of Scopus and Web of Science in locating corrections within scholarly journals, tools that did not exist (Scopus) or were not utilized (Science Citation Index) in the earlier studies.

\section{METHODOLOGY}

The study involved selecting ten multidisciplinary chemistry journals, obtaining total article and correction counts, and then quantitatively/qualitatively analyzing corrections for one chemistry journal in detail. A six-year period from 2000 to 2005 was selected for this study to provide a meaningful but manageable amount of data over a recent time period.

There are several resources for identifying core chemistry journals. These include Journal Citation Reports, CAS Source Index, and Ulrich's International Periodicals (Maizell 1998). For this study, a list of general chemistry journals was obtained using the "ChemistryMultidisciplinary" category within Journal Citation Reports, which provided chemistry journals "having a general or interdisciplinary approach to the chemical sciences" (Thomson Reuters 2009). The top ten multidisciplinary chemistry journals for 2005 were selected from the "Chemistry-Multidisciplinary" category based on impact factor.

For this study, an article will be defined as any content within a journal that is typically indexed by databases (e.g., articles, communications, editorials, published corrections, book reviews, etc.) to avoid awkward or confusing terminology throughout the remainder of the paper.

The total number of articles within each of the ten journals for 2000-2005 was obtained using both Scopus and Web of Science. The total number of published corrections within the ten 
journals was determined and compared using three methods: Scopus, Web of Science, and consulting the actual print and/or online journals. Medline was not included in this study since it indexed only eight of the ten chemistry journals and did so selectively (National Library of Medicine 2005). Corrections were obtained from Scopus by selecting “Erratum” under Document Type, entering the journal title as the Source Title, and selecting 2000 and 2005 as the Date Range. In Web of Science, corrections were obtained by selecting "Correction" and “Correction, Addition" under Document Type, entering the journal title as the Publication Name, and 2000-2005 as the Year Published. The print and/or online versions of the ten journals were also examined to obtain the actual number of corrections and retractions. Since both databases index retractions as a type of Erratum or Correction, the remainder of this paper will also treat retractions as a type of correction.

A correction rate was determined by dividing the actual number of corrections obtained from the print and/or online sources by the total number of articles as determined by Web of Science. The rationale for selecting Web of Science over Scopus for the total number of articles will be discussed in the" Results and Discussion" section of this article.

The quantitative and qualitative nature of corrections within the chemical literature was studied in more detail using the Journal of the American Chemical Society, a major chemistry journal publishing original research articles. Each of the 220 corrections that the Journal of the American Chemical Society published between 2000 and 2005 was examined and then categorized based on the type of correction (or error). The bibliographic information for each correction was downloaded from Web of Science and entered into a spreadsheet to manage the bibliographic and other data collected during the study. While there is always some subjectivity when developing qualitative categories, many clearly defined categories emerged through an 
iterative process of combining and redefining. Clearly defined categories and descriptions were developed to ensure integrity of the categorizing process and distinctions among the various types of corrections. In many cases, original articles were consulted to clarify and understand the correction being made.

There were often more than one correction per published correction (e.g., wrong formula and omitted coauthor) and in some cases the corrections were related (e.g., wrong formula and wrong data value). The latter would also count as two separate corrections since the errors may be independent; that is, it could be a wrong formula but the incorrect data could be a result of a typographical rather than computational error. Each of the 220 published corrections was then assigned to one or more categories.

The number of individual corrections per published correction was determined by summing the number of individual corrections in each published correction. The average number of individual corrections per published correction was calculated by dividing the total number of individual corrections by the 220 published corrections. The length of time between publication of the original article and the correction was calculated using the Web publication dates.

Since methodologies and data collection varied among Addelston and Goldsmith (1966), Sabine (1985) and this study, it is difficult to compare correction rates for the Journal of the American Chemical Society over that time period. To compare correction rates over time, correction rates were calculated for each year from 1965 to 2005 based on data from Web of Science.

Finally, an assessment was made regarding the linking of 220 original articles to their corrections within the American Chemical Society e-journal platform. This was accomplished by 
checking the 220 original articles at the Journal of the American Chemical Society website for information or links to the correction.

\section{RESULTS AND DISCUSSION}

The top ten multidisciplinary chemistry journals as defined by 2005 Journal Citation Reports are presented in Table 1. The top ten publications consist of six research journals, three review journals, and one serial monograph comprised of review articles. For the sake of readability, all ten publications will be referred to as journals for the remainder of the paper. Table 1 also

TABLE 1 . Article and correction counts for the top ten multidisciplinary chemistry journals ${ }^{\mathrm{a}}, 2000-2005$.

\begin{tabular}{|c|c|c|c|c|c|c|}
\hline \multirow{2}{*}{ Journal Title } & \multicolumn{2}{|c|}{ All Publication Types ${ }^{b}$} & \multicolumn{3}{|c|}{ Corrections } & \multirow{2}{*}{$\begin{array}{l}\text { Correction } \\
\text { Rate }(\%)\end{array}$} \\
\hline & Scopus & WOS $^{c}$ & Actual & Scopus & WOS $^{\mathrm{c}}$ & \\
\hline Accounts of Chemical Research & 650 & 652 & 5 & 5 & 5 & 0.8 \\
\hline Angewandte Chemie Int. Ed. & & 6,915 & 150 & 138 & 110 & 2.2 \\
\hline Chemical Communications & 8,270 & 8,275 & 141 & 137 & 104 & 1.7 \\
\hline Chemical Reviews & 921 & 919 & 15 & 7 & 15 & 1.6 \\
\hline Chemical Society Reviews & & 327 & 6 & 3 & 5 & 1.8 \\
\hline Chemistry A European Journal & 3,676 & 3,569 & 59 & 47 & 42 & 1.7 \\
\hline Journal of the American Chem & 17,054 & 17,067 & 220 & 217 & 219 & 1.4 \\
\hline Lab on a Chip & 457 & 470 & 5 & 4 & 5 & 1.1 \\
\hline Nano Letters & 1,757 & 1,711 & 16 & 12 & 15 & 0.9 \\
\hline Topics in Current Chemistry & $138^{\mathrm{d}}$ & 386 & 4 & 0 & 0 & 1.0 \\
\hline TOTAL & & & 621 & 570 & 520 & \\
\hline
\end{tabular}

${ }^{\text {a }}$ As ranked by 2005 journal Citation Reports.

${ }^{\mathrm{b}}$ Articles, communications, editorials, published corrections, book reviews, etc.

${ }^{c}$ Web of Science.

${ }^{\mathrm{d}}$ Scopus started indexing this publication in 2005.

contains a summary of the total number of articles indexed by Scopus and Web of Science, as well as the number of actual and indexed published corrections for the ten journals. 
The correction rates were calculated by dividing the number of actual published corrections by the total number of articles as determined by Web of Science for a particular journal. Web of Science was chosen for the total article count, because it had a higher total article count for most of the journals studied. Using Scopus, Web of Science, or an average of the two values would not have changed the correction rates significantly for most of the journals. A better approach would be to count the total number of articles based on print versions of the journals, but this was deemed to be too tedious and possessed its own set of challenges (e.g., errors associated with counting over 40,000 publications and lack of access to some print volumes).

The correction rate is $1.4 \pm 0.5$ percent and ranged from 0.8 percent to 2.2 percent (Table 1). This is slightly lower than the 2.3 percent error rate reported by Sabine (1985), though he focused primarily on biological literature, examined only one year of data, and excluded all publication types except research articles from this error rate calculation. Aside from a fairly consistent correction rate among the ten journals, one of the more interesting findings is that not all published corrections in the ten journals are indexed by Scopus or Web of Science. If one excludes the Topics in Current Chemistry corrections, which neither of the databases indexed, Web of Science was closer to the correct number of actual corrections for five of the nine other publications. On the other hand, Scopus identified more of the total corrections, 567 (91.3 percent) versus 520 (83.7 percent), for all ten journals. Both databases omitted a large number of corrections from three journals in particular: Angewandte Chemie International Edition, Chemical Communications, and Chemistry A European Journal.

The headings used to identify corrections in the tables of contents vary within and among the ten journals studied. Corrections are typically identified using fairly common terminology (e.g., additions and corrections, corrigenda, and errata), but occasionally more ambiguous terms were 
used (e.g., addendum, apologies, etc.). Regardless of the terminology, Scopus indexed all of these as Erratum and Web of Science indexed them as Correction (or Correction, Addition). While the varying terminology might cause some difficulty with locating corrections, it does not appear to be the major cause of omissions. Of the 152 omitted corrections from both Scopus (51) and Web of Science (101), only nine corrections were omitted from both databases. Since there is little overlap, it suggests database indexing issues rather than a problem with how the corrections are presented in the journals. It appears that the omitted corrections were simply overlooked.

The specific indexing practices of Scopus and Web of Science for materials published between 2000 and 2005 were not investigated, but using online versions of print journals can be problematic. In the case of the online version of Chemical Communications, "Additions \& corrections" were placed in a pdf file titled "Back Matter" for 2004-2005, identified only in the tables of contents for 2002-2003, and completely omitted from 2000 to 2001. In this particular case, print and online versions of the journal are not equivalent and this could have implications for indexing corrections or locating published corrections in the online journal. This also has implications for reference services and collection management, especially if print volumes are stored remotely or discarded.

The numbers of corrections obtained from Scopus and Web of Science are just counts and do not necessarily mean that the bibliographic information for each individual record is correct. Aside from omitting corrections, those that were included were not always properly indexed (e.g., incorrect volume/issue numbers, titles, and authors). Of the 104 corrections obtained from Web of Science for Chemical Communications, six were duplicate records. While Scopus had more accurate counts for Angewantde Chemie International Edition and Chemical 
Communications, over 100 corrections lacked any kind of descriptive title referring to the original article title and many lacked authors as well. It is standard for both databases to include the original title and the author(s) within the indexed correction. Without a title and author, it would be difficult to discover these corrections unless searching for corrections within a specific journal. Web of Science provided more complete and descriptive records for the corrections.

TABLE 2. Number of individual corrections per published correction for the Journal of the American Chemical Society, 2000-2005.

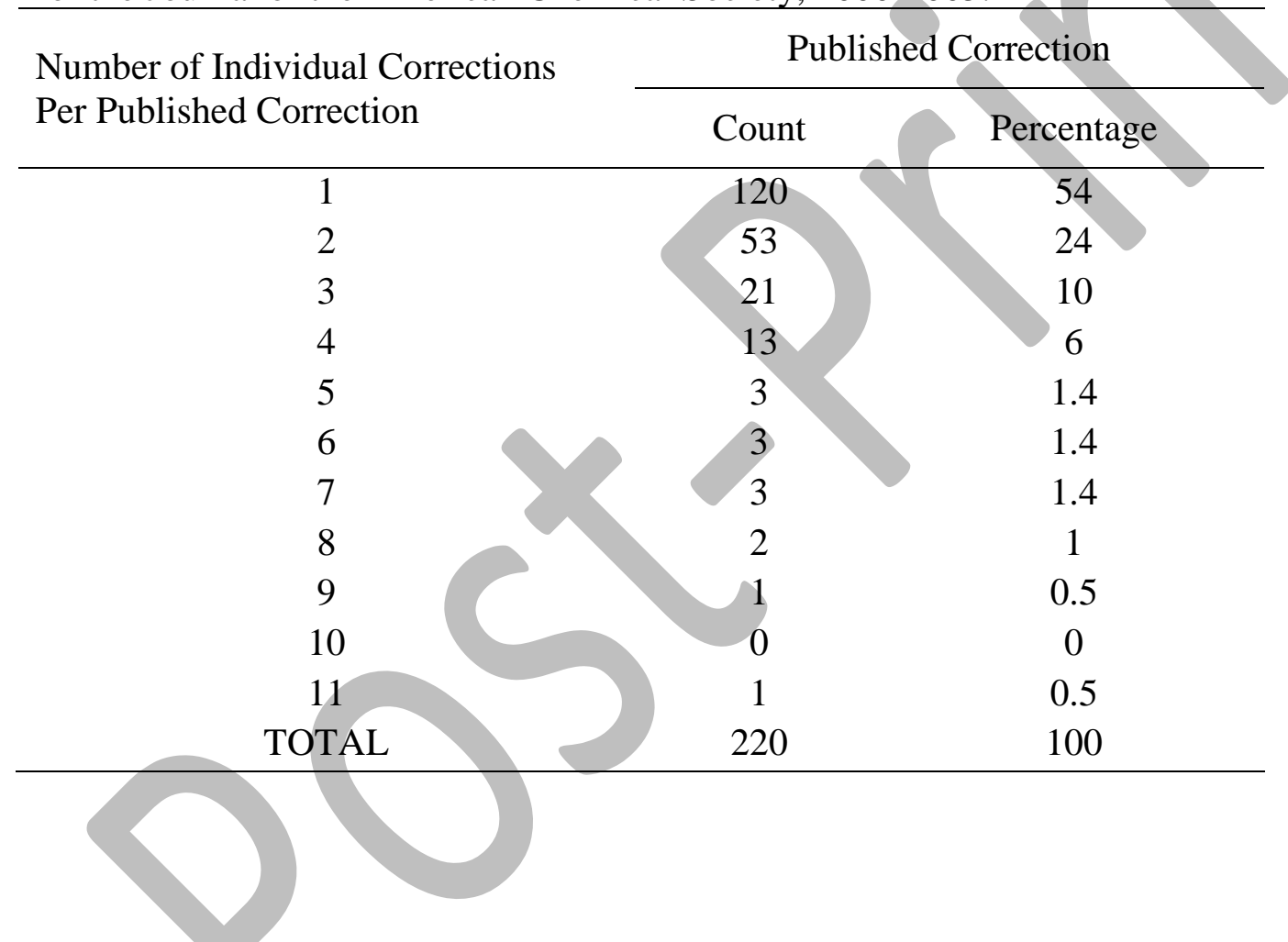

The Journal of the American Chemical Society published 220 corrections for 2000-2005. The number of individual corrections per published correction is presented in Table 2. Almost half (46 percent) of the 220 published corrections contained two or more individual corrections. The number of individual corrections per published correction ranged from 1 to 11 . The 220 published corrections contained a total of 431 individual corrections. After examining the 431 
corrections, 18 categories of corrections (or types of errors) were created and the corrections

were categorized. The 18 categories and their descriptions are presented in Table 3.

TABLE 3. Categories of corrections and their descriptions.

\begin{tabular}{|c|c|}
\hline Category & Description \\
\hline Addition & Added additional material and/or clarification. \\
\hline Author & $\begin{array}{l}\text { Misspelling of an author's name, changing the order of coauthor names, and/or } \\
\text { correcting author affiliation. }\end{array}$ \\
\hline Chemical & Incorrect chemical name, including those in tables and figures. \\
\hline Conclusion & Major claim or conclusion in the paper is determined to be incorrect. \\
\hline Data & $\begin{array}{l}\text { Incorrect experimental, calculated, or interpreted values (e.g., NMR peaks), as } \\
\text { well as constants, units of measure, and sign errors. Excludes data presented in } \\
\text { figures and tables. See Figure/Table Data. }\end{array}$ \\
\hline Equation & Incorrect mathematical or chemical equation. \\
\hline Figure & $\begin{array}{l}\text { Incorrect figure. Includes errors associated with labels/headings, duplicate } \\
\text { figures, captions, and omitted figures. }\end{array}$ \\
\hline Figure/Table Data & $\begin{array}{l}\text { One or more instances of incorrect data within a figure or table. Systematic errors } \\
\text { are only counted once (e.g., omission of a minus "-"sign or off by a factor of } 10 \\
\text { for } 20 \text { values in a table). Includes incorrect experimental, calculated, or } \\
\text { interpreted values (e.g., NMR peaks), as well as constants, units of measure, and } \\
\text { sign errors. }\end{array}$ \\
\hline Formula & $\begin{array}{l}\text { Incorrect chemical formula including those in tables and figures. Also includes } \\
\text { DNA and peptide sequences, as well as charges on ions. }\end{array}$ \\
\hline Omission-Acknowledgmen & Omission of an individual or organization in the acknowledgement. \\
\hline Omission-Author & Omission of an author's name. \\
\hline Omission-Reference & Omission of a reference. \\
\hline Reactant-Proc & $\begin{array}{l}\text { Incorrect amounts or concentrations of reactants/catalysts, as well as product } \\
\text { yields. }\end{array}$ \\
\hline & Wrong or incomplete references. Includes both in-text and footnoted references. \\
\hline Rephrase & A portion of the text is rephrased, edited, or deleted. \\
\hline Retraction & Author(s) states that the paper is to be retracted. \\
\hline Structure & $\begin{array}{l}\text { Incorrect chemical structure, including ball-and-stick and computer generated } \\
\text { models. Each structure is counted separately, including those in tables and } \\
\text { figures. Includes structures that were omitted. }\end{array}$ \\
\hline Supporting Information & $\begin{array}{l}\text { Includes supporting information that was omitted, inaccessible online, or entirely } \\
\text { replaced. }\end{array}$ \\
\hline
\end{tabular}


Some of the eighteen categories could be combined, but a decision was made to retain as much granularity as possible while maintaining a reasonable number of categories. For example, Data and Figure/Table Data represent similar yet different types of corrections. A Data correction refers to specific data errors within the text, whereas Figure/Table Data corrections are data errors within figures and tables. The latter may represent multiple systematic errors (e.g., omission of a minus “-" sign or off by a factor of 10 for all values in a table). In some ways this category underestimates the number of corrections, but in a few instances there were numerous errors within tables due to this type of systematic error. To count each separately would have grossly overestimated the number of corrections and problems. Another example is Author and Omission-Author. While these categories could be combined, the former represents errors associated with the author's name and/or correcting affiliation. On the other hand, OmissionAuthor is used when a coauthor has been omitted from a paper. While the result could be similar from a bibliographic standpoint, especially for database searching, these are two distinct types of errors, and a decision was made to retain separate categories.

The number of corrections by category for the 431 individual corrections is presented in Figure 1. Corrections associated with structures were the most common. The second most common was omissions of relevant references. Data corrections ranked third, though a combination of Data and Figure/Table Data corrections would have exceeded corrections associated with structures. Regardless of their exact ranking, it is clear that a large percentage of the corrections are associated with a failure to cite relevant literature. Approximately 25 (or 11 percent) of published corrections thanked another researcher for pointing out the error(s) in 


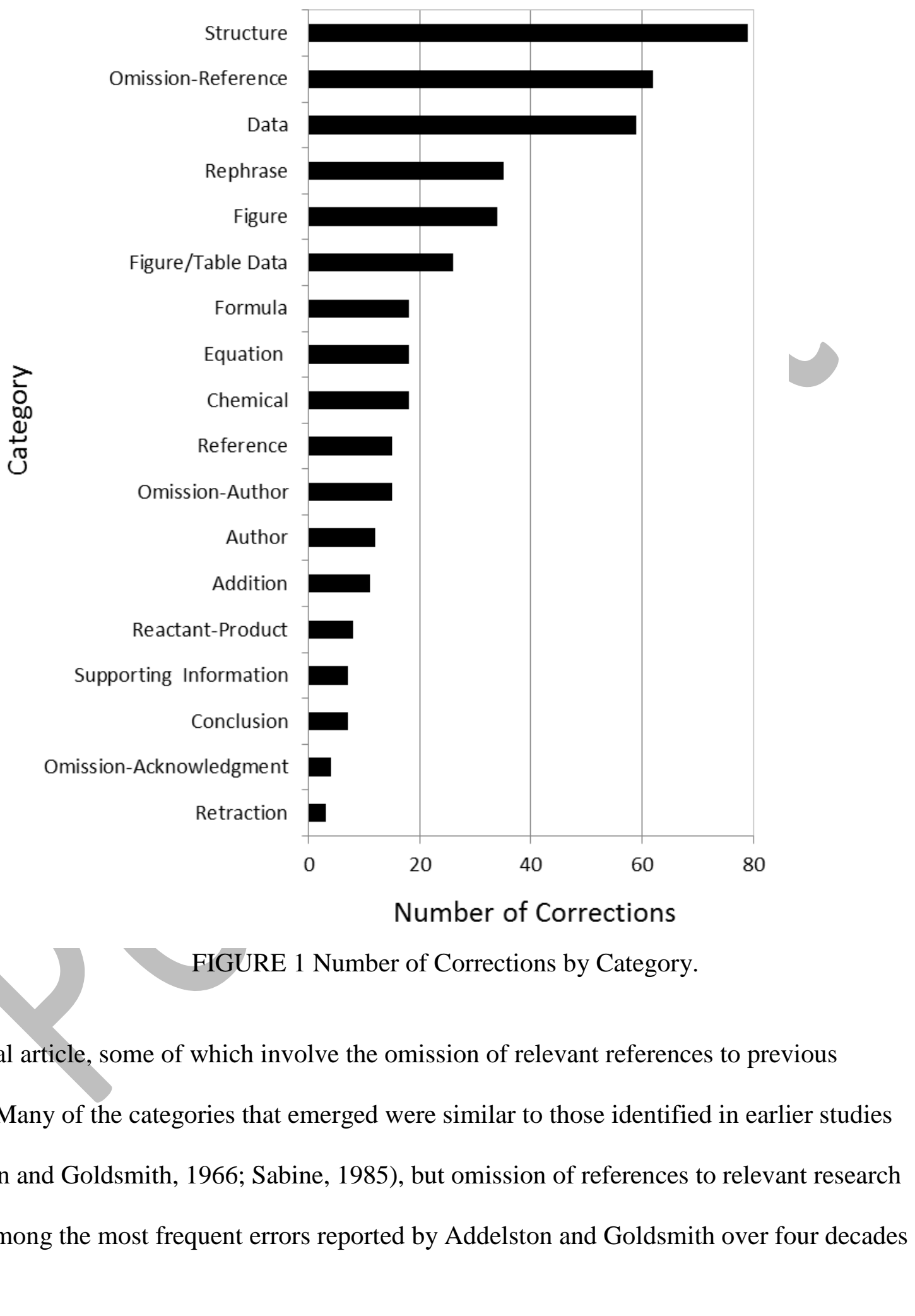


The average time between publication of the original article and correction was $10 \pm 19$ months. Such a large standard deviation is understandable considering that the range is 0.5 to 187 months. The median time between publication of the article and correction, which is 5 months, is much more informative. A frequency chart (Figure 2) presents the time between publication of the original article and correction. More than half of the corrections (54 percent) were published within 6 months of the original article; however, more than 20 percent were published over a year later.

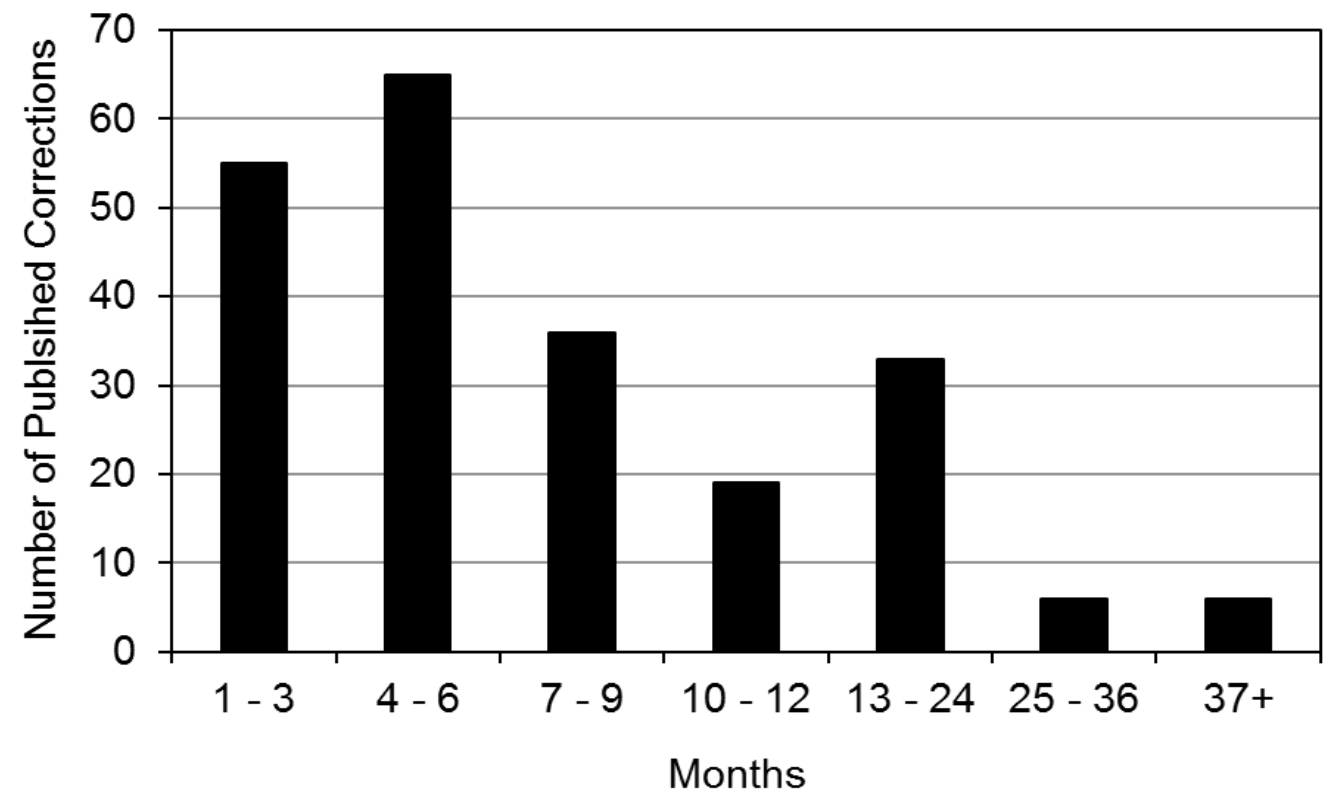

FIGURE 2. Frequency Chart Presenting Time between Publication of Orginal Article and Correction.

Considering the length of time between original article and published correction, one may wonder about the effectiveness of published corrections in terms of maintaining the integrity of the scholarly record, especially if the corrections are not linked in an online environment as Poworoznek (2003) reported. Of the 220 published corrections, 81 corrections have been cited two or more times since 2000 . The 220 corrections have been collectively cited 421 times with 
an average citation rate of 1.8 . One correction has been cited 28 times. So the corrections are being found and cited, but a study similar to that of Devlin (1969) would be required to assess the true effectiveness of published corrections. One would need to examine how every citation to the original article with a published correction was cited and whether the error(s) was either corrected, avoided, or immaterial to the citing article.

Figure 3 shows the correction rates for the Journal of the American Chemical Society from 1965 to 2005 . Over this period of time, correction rates have consistently decreased. The trend is statistically significant based on a correlation coefficient of 0.6418 and a p-value of $<.0001$.

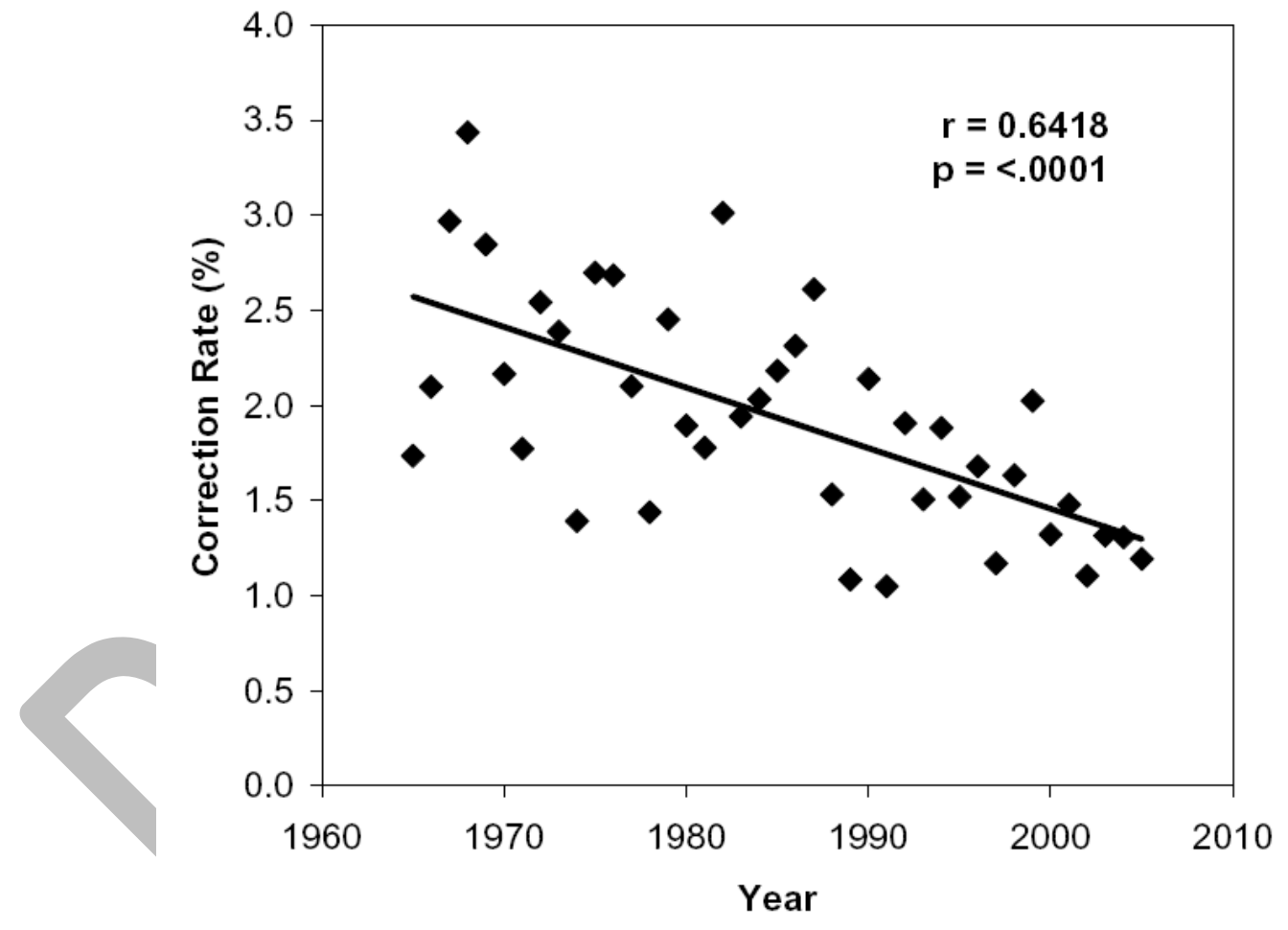

FIGURE 3. Correction Rates for the Journal of the American Chemical Society from 1965-2005.

The reason for this decrease requires further study. It could be due to fewer author errors, increased reluctance to publish corrections, or some other factors. Consequently, it is difficult to say whether the decrease in correction rate is a positive development at this point. 
Approximately 96 percent of the 220 original articles were linked to their corrections in the American Chemical Society e-journal platform. The link to the pdf containing the "addition/correction" is in close proximity to the full-text pdf, though it is not very prominently displayed. Considering that Poworoznek (2003) found no links from the original article to their corrections for the Journal of the American Chemical Society, it appears that the situation has improved considerably for at least this one journal.

\section{CONCLUSION}

A number of tools and approaches are available to locate corrections within the chemical literature. In addition to the published corrections in journals, Scopus and Web of Science facilitate the discovery of corrections during searches. Both databases were successful in identifying the majority of corrections, though both had their share of omissions and errors. The average correction rate among the ten journals studied was $1.4 \pm 0.5$ percent, meaning one could expect to find 1-2 corrections for every 100 chemistry articles. While the correction rate for the Journal of the American Chemical Society has consistently decreased since 1965, it is unclear whether that trend represents a decrease in errors, increased reluctance to publish corrections, or some other factors. Among published corrections, chemical structures and various data errors were the most common; however, omission of relevant references was significant and appears to have increased over the years. This provides an opportunity to increase information literacy not only by educating users on how to locate relevant references to previous work but also on how to identify and locate corrections that are essential to understanding articles, reproducing experiments, and maintaining the integrity of the scholarly record. Errors will never be 
completely eliminated, but having knowledge of their type and frequency can assist authors in preventing such errors.

While linking of corrections to the original article appears to have improved for at least one journal, there is still an opportunity to ensure that all published corrections are linked to the original articles and displayed in a more prominent manner. Scopus and Web of Science could also improve on their indexing of corrections in terms of accuracy and completeness. Both database vendors could add considerable value to their databases by inserting a prominent link from the original article record to the correction record rather than treating the correction as just another citation.

\section{REFERENCES}

Addelston, Aaron, and Uriel J. Goldsmith. 1966. Procedures for detecting errors in chemical literature. Journal of Chemical Documentation 6 (3): 126-129.

Atlas, Michel C. 2004. Retraction policies of high-impact biomedical journals. Journal of the Medical Library Association 92 (2): 242-250.

Budd, John M., MaryEllen Sievert, Tom R. Schultz, and Caryn Scoville. 1999. Effects of article retraction on citation and practice in medicine. Bulletin of the Medical Library Association 87 (4): 437-443.

Colaianni, Lois A. 1992. Retraction, comment, and errata policies of the US National Library of Medicine. Lancet 340: 536-537.

Devlin, Patricia A. 1969. The effectiveness of errata sheets in preventing error transmission in the English-language chemical literature. Philadelphia: Drexel Institute of Technology.

Garfield, Eugene. 1963. Science citation index. Science Citation Index 1961 1: v-xvi.

Garfield, Eugene. 1987. How to avoid spreading error: Scientists must search for corrections. The Scientist 1 (19): 9.

Garfield, Eugene, and Alfred Welljams-Dorof. 1990. The impact of fraudulent research on the scientific literature: The Stephen E. Breuning case. JAMA 263 (10): 1424-1426.

Jones, Dixie, A., Michael M. Watson, Marianne Comegys, Alice Burnett, and Betty Tucker. 2003. Errata and retractions in electronic journals: Notification practices. Journal of Hospital Librarianship 3 (2): 19-27.

Kotzin, Sheldon, and Peri L. Schuyler. 1989. NLM's practices for handling errata and retractions. Bulletin of the Medical Library Association 77 (4): 337-342.

Loepprich, Joyce C. 1973. Errata control in biomedical journals. Bulletin of the Medical Library Association 61 (3): 319-323. 
Maizell, Robert E. 1998. How to find chemical information: A guide for practicing chemists, educators, and students. 3rd ed. New York: John Wiley \& Sons.

Nath, Sara B., Steven C. Marcus, and Benjamin G. Druss. 2006. Retractions in the research literature: misconduct or mistakes? Medical Journal of Australia 185 (3): 152-154.

National Library of Medicine. 2005. List of journals indexed for Medline. Bethesda: U.S. Dept. of Health and Human Services.

Pfeifer, Mark P., and Gwendolyn L. Snodgrass. 1990. The continued use of retracted, invalid scientific literature. JAMA 263 (10): 1420-1423.

Poworoznek, Emily L. 2003. Linking of errata: Current practices in online physical sciences journals. Journal of the American Society for Information Science and Technology 54 (12): 1153-1159.

Redman, B. K., H. N. Yarandi, and J. F. Merz. 2008. Empirical developments in retraction. Journal of Medical Ethics 34: 807-809.

Sabine, John R. 1985. The error rate in biological publication: A preliminary survey. Science, Technology, \& Human Values 10 (1): 62-69.

Thomson Reuters. 2009. Scope notes. Journal Citation Reports. http://adminapps.isiknowledge.com/JCR/static_html/scope_notes/SCIENCE/2008/SCOPE_SCI.htm (accessed June 2, 2009). 